\title{
Structure of CdS-arachidic acid composite LB multilayers
}

\author{
N. Prasanth Kumar a, S.N. Narang ${ }^{a}$, S. Major ${ }^{a, *}$, Satish Vitta ${ }^{b}$, S.S. Talwar ${ }^{c}$, \\ P. Dubcek ${ }^{\mathrm{d}}, \mathrm{H}$. Amenitsch ${ }^{\mathrm{d}}$, S. Bernstorff ${ }^{\mathrm{d}}$ \\ a Department of Physics, Indian Institute of Technology, Bombay, Mumbai 400076, India \\ ${ }^{\mathrm{b}}$ Department of Metallurgical Engineering and Materials Science, Indian Institute of Technology, Bombay, Mumbai 400076, India \\ ${ }^{\mathrm{c}}$ Department of Chemistry, Indian Institute of Technology, Bombay 400076, Mumbai, India \\ ' Sincrtrone Trieste, Basovizza, Italy
}

\begin{abstract}
Langmuir-Blodgett (LB) multilayers of cadmium arachidate were used as precursors to grow semiconducting CdS nanoclusters. The formation of $\mathrm{CdS}$ in the multilayers was determined by Fourier transform-infrared (FT-IR), ultraviolet-visible (UV-vis) and Raman spectroscopy. The structural changes occurring as a consequence of CdS formation have been characterized using X-ray reflection (XR) and grazing incidence X-ray diffraction (GIXD) techniques. The CdS containing composite multilayers exhibit the presence of two types of molecular domains, one with close packed herringbone arrangement and the other with tilted molecular chains with no in-plane order. The structural and spectroscopic evidences together suggest that the CdS nanoclusters formed within the arachidic acid LB matrix are quasi two-dimensional in nature with lateral dimension $\sim 5-10 \mathrm{~nm}$ and thickness $\sim 1.1 \mathrm{~nm}$.
\end{abstract}

Keywords: Langmuir-Blodgett; Nanoclusters; CdS; Molecular packing

\section{Introduction}

Langmuir-Blodgett (LB) multilayers are currently being used as precursors for the growth of semiconducting nanoclusters within the layered matrix through post deposition treatment [1-3]. The interest in this approach is primarily because the layered structure and molecular order present in the LB multilayers are expected to help in achieving better control over the size, shape and distribution of nanoclusters. LB multilayers of divalent fatty acid salts like cadmium arachidate/ stearate have been most extensively used to develop and understand the growth process of semiconducting chalcogenide (e.g. CdS) nanoclusters [4-10]. However, inspite of the extensive work on the $\mathrm{CdS}$-fatty acid system, the issue of size and shape of CdS nanoclusters still remains to be completely understood. In most of the early work, the particle size has been usually estimated from the shift of the absorption onset and/or excitonic peak position assuming spherical shape 
of nanoclusters [5-8]. However, more recent approaches using techniques like grazing incidence X-ray scattering/diffraction, spectroscopic ellipsometry and Raman spectroscopy have shown that the assumption of spherical nanoparticles may not be true [11-14]. X-ray scattering/diffraction studies have shown that the CdS nanoclusters grow as thin sheets [11] or as quasi two-dimensional discs [13] at the headgroup interfaces while Raman studies have shown that the CdS nanoclusters could be ellipsoidal [14] in shape. On the other hand, transmission electron microscopy [3,15] and Rutherford back-scattering [16] studies have supported the spherical particle picture. There are also other issues such as the kinetics of $\mathrm{CdS}$ formation, growth and organization of nanoclusters within the LB matrix and the overall structure of the resulting composite multilayers, which need to be addressed. These issues have important technological implications for the practical use of these nanocomposite systems in novel nanostructured devices.

In the present work, cadmium arachidate (CdA) multilayers have been used to develop CdS nanoclusters in the LB matrix through $\mathrm{H}_{2} \mathrm{~S}$ exposure. The formation of $\mathrm{CdS}$ nanoclusters and the resulting changes in the overall structure of the composite multilayer as a function of $\mathrm{H}_{2} \mathrm{~S}$ exposure have been investigated using X-ray reflectivity (XR), grazing incidence X-ray diffraction (GIXD), ultraviolet-visible (UV-vis), Fourier transform-infrared (FT-IR) and Raman spectroscopic techniques. Based on the combined structural and spectroscopic evidences the issue of size and shape of $\mathrm{CdS}$ nanoclusters has been studied in detail.

\section{Experimental}

The precursor cadmium arachidate LB multilayers were deposited using a KSV-3000 LB instrument kept in a clean room. Arachidic acid (Aldrich 99\%) and chloroform (HPLC grade) were used for the preparation of the spreading solution $\left(1 \mathrm{mg} \mathrm{ml}^{-1}\right)$. Deionized and Millipore ultrafiltered water (resistivity, $18.2 \mathrm{M} \Omega \mathrm{cm}$ ) was used for preparing the subphase. A $4 \times 10^{-4} \mathrm{M}$ solution of anhydrous $\mathrm{CdCl}_{2}$ was added to the subphase and $\mathrm{pH}$ was adjusted to 6.5 , by adding $\mathrm{NaHCO}_{3}$. Quartz and $\mathrm{CaF}_{2}$ were used as substrates and were cleaned using standard procedures. The multilayer depositions were carried out at a constant surface pressure of $30 \mathrm{mN} \mathrm{m}^{-1}$. The compressed monolayer was transferred by vertical dipping method at a speed of $3 \mathrm{~mm} \min ^{-1}$. Typically, 25 monolayers were transferred in each case. The LB multilayers were exposed for different durations to a constant flow of $\mathrm{H}_{2} \mathrm{~S}$ gas generated using the Kipp's apparatus. Exposures of duration larger than $1 \mathrm{~h}$ were carried out in steps of $1 \mathrm{~h}$. UV-vis spectra were obtained with a Shimadzu UV-160A spectrophotometer and FTIR studies were carried out with a Nicolet (Impact-400 model) instrument. XR studies were performed with Philips PW 1820 powder diffractometer using $\mathrm{Cu}-\mathrm{K}_{\alpha}$ radiation in the $2 \theta$ range $4-20^{\circ}$. The GIXD studies were performed using synchrotron radiation of wavelength $0.155 \mathrm{~nm}$ at the Italian synchrotron source ELETTRA (SAXS beamline). An asymmetrically cut $\mathrm{Si}(111)$ double crystal both selects and monochromates the 0.155 $\mathrm{nm}$ radiation. A guard slit of size $2.0 \times 0.05 \mathrm{~mm}$ $(H \times V)$ defines the illuminated area on the sample surface. A one-dimensional gas filled detector, which has an angular resolution of $0.05^{\circ}$ was used to detect the diffracted beam whose size was defined by a $1.6 \times 0.6 \mathrm{~mm}$ exit slit. The angle of incidence was kept close to the critical angle for total reflection, $\sim 0.3^{\circ}$ and the diffraction spectrum was studied in the range $15-35^{\circ}$. Raman spectra were recorded in the back scattering geometry using an argon ion laser with standard detection and data collection systems. Resonance conditions could be obtained with the $457.9 \mathrm{~nm}$ line of the argon laser and was used with a laser power of $\leq 50 \mathrm{~mW}$. The slit-width was kept at $250 \mu \mathrm{m}$ and the data was collected for $20 \mathrm{~s}$ in order to get relatively good signal-to-noise ratio.

\section{Results and discussion}

The changes in the composition of the precursor CdA multilayer have been studied as function of exposure to $\mathrm{H}_{2} \mathrm{~S}$ gas using FT-IR and UV-vis 
spectroscopies. Fig. 1 shows the FT-IR spectra of the CdA multilayer in the as-deposited state and after $\mathrm{H}_{2} \mathrm{~S}$ exposures of $15 \mathrm{~min}, 1$ and $2 \mathrm{~h}$. The as-deposited multilayer (curve (a)) exhibits strong absorption band at $\sim 1540 \mathrm{~cm}^{-1}$ due to asymmetric carbonyl stretching vibration of the carboxylate group, indicating that the transferred multilayers are present in the form of pure arachidate salt. After $15 \mathrm{~min}$ of $\mathrm{H}_{2} \mathrm{~S}$ exposure, the spectrum (curve (b)) exhibits the appearance of an

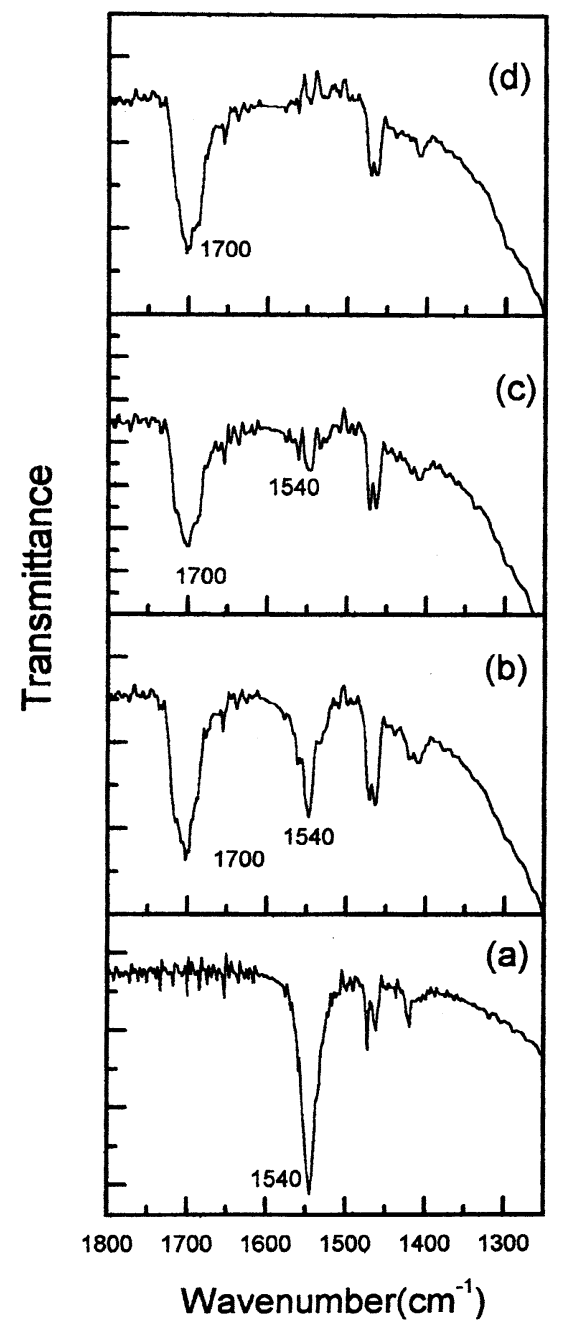

Fig. 1. FTIR spectra of a CdA LB multilayer in (a) the as-deposited state and after $\mathrm{H}_{2} \mathrm{~S}$ exposure for (b) $15 \mathrm{~min}$, (c) 1 $\mathrm{h}$ and (d) $2 \mathrm{~h}$, showing the arachidate salt to arachidic acid conversion process.

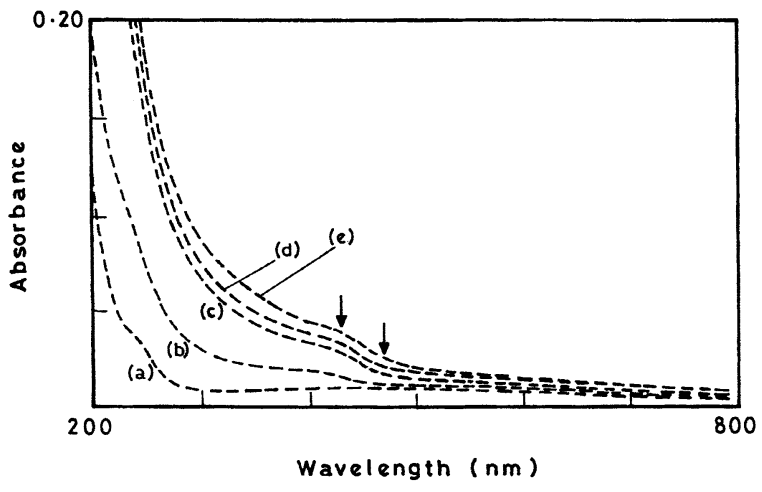

Fig. 2. UV-vis absorption spectra of the CdA multilayer in (a) as-deposited state and after $\mathrm{H}_{2} \mathrm{~S}$ exposed for (b) $15 \mathrm{~min}$, (c) 1 $\mathrm{h}$, (d) $2 \mathrm{~h}$ and (e) $3 \mathrm{~h}$. The enhanced absorption below $500 \mathrm{~nm}$ indicates the formation of $\mathrm{CdS}$ in the arachidic acid matrix.

additional absorption band $\sim 1700 \mathrm{~cm}^{-1}$ due to carbonyl stretching vibration of the carboxylic acid group indicating the beginning of the conversion of arachidate salt into arachidic acid. After 1 $\mathrm{h}$ of $\mathrm{H}_{2} \mathrm{~S}$ exposure, the spectrum (curve (c)) exhibits absorption bands both at $\sim 1540$ and $\sim$ $1700 \mathrm{~cm}^{-1}$ indicating the presence of a mixture of arachidate salt and arachidic acid. However, after $2 \mathrm{~h}$ of $\mathrm{H}_{2} \mathrm{~S}$ exposure (curve (d)), the absorption band at $\sim 1540 \mathrm{~cm}^{-1}$ disappears completely and only a strong absorption band at $\sim 1700 \mathrm{~cm}^{-1}$ was observed indicating complete conversion of arachidate salt to arachidic acid. The chemical conversion of the salt to acid is indicative of the formation of $\mathrm{CdS}$ in the multilayers. The FT-IR spectra show no changes on longer exposures $(>2 \mathrm{~h})$ indicating a saturation behavior of the salt to acid conversion process.

Fig. 2 shows the UV-vis absorption spectra of $\mathrm{CdA}$ multilayers exposed to $\mathrm{H}_{2} \mathrm{~S}$ gas for different durations. Curve (a) corresponds to the as-deposited CdA multilayer. The UV-vis absorption behavior after $15 \mathrm{~min}$ exposure is shown by curve (b) and it exhibits an increase in absorbance below $\sim 450 \mathrm{~nm}(2.7 \mathrm{eV})$ when compared with the as-deposited multilayer. The enhanced absorbance is attributed to the presence of CdS in the LB matrix. The spectrum also exhibits a small broad peak at $\sim 400 \mathrm{~nm}$, which is attributed to the excitonic band of $\mathrm{CdS}[17,18]$. On increasing the exposure duration to $1 \mathrm{~h}$ (curve (c)), $2 \mathrm{~h}$ (curve 
(d)) and $3 \mathrm{~h}$. (curve (e)), an increase in the overall absorbance below $450 \mathrm{~nm}$ was progressively observed. The position of the absorption onset ( $\sim$ $460 \mathrm{~nm})$ and the excitonic band $(\sim 430 \mathrm{~nm})$ were also found to shift slightly towards longer wavelengths in these cases. No further change in the UV-vis spectra was observed for exposure durations greater than $3 \mathrm{~h}$. The absorption onset at $\sim 460 \mathrm{~nm}$ in the CdS containing multilayer is blue shifted as compared with bulk CdS absorption edge at $515 \mathrm{~nm}$. The blue shift of the absorption onset and the presence of excitonic band indicate size quantitation effects associated with the formation of $\mathrm{CdS}$ in the arachidic acid matrix [17].

Fig. 3(a) shows the XR pattern for the as-deposited CdA multilayer. The XR pattern exhibits well defined Bragg peaks corresponding to $(00 l)$ reflections upto 11 orders indicating the presence of ordered layered structure with an average bilayer period of $5.5 \mathrm{~nm}$. The bilayer period of 5.5 nm corresponds to alkyl chains packed nearly perpendicular to the layer plane. The XR pattern after $1 \mathrm{~h}$ of the $\mathrm{H}_{2} \mathrm{~S}$ exposure (Fig. 3(b)) shows just an overall decrease in the intensity of the Bragg peaks as compared with the as-deposited multilayer, indicating a decrease in layered structural order as a result of $\mathrm{H}_{2} \mathrm{~S}$ exposure. Fig. 3(c and d) show the XR patterns of the CdA multilayer after 3 and $5 \mathrm{~h}$ of $\mathrm{H}_{2} \mathrm{~S}$ exposure, respectively. An additional set of Bragg peaks develops after $3 \mathrm{~h}$ exposure which become prominent in the $5 \mathrm{~h}$ exposed multilayer. These Bragg peaks have been indexed as $\left(003^{\prime}\right)$ and $\left(005^{\prime}\right)$ reflections of the new type of layered structure which has a bilayer period of $4.4 \mathrm{~nm}$. A bilayer period of $4.4 \mathrm{~nm}$ corresponds to packing of molecules with alkyl chains tilted at an angle of $\sim 37^{\circ}$ with respect to the layer normal.

Fig. 4 shows the GIXD patterns for the CdA multilayers exposed to $\mathrm{H}_{2} \mathrm{~S}$ gas for different durations. The GIXD pattern for the as-deposited CdA multilayer shown in Fig. 4(a) exhibits diffraction peaks corresponding to (11) and (02) reflections of a centred rectangular two-dimensional lattice [19]. The lattice parameters obtained are, $a=0.47 \mathrm{~nm}$ and $b=0.72 \mathrm{~nm}$, which are in close agreement with the layer cell dimensions of an ideal close packed herringbone structure,

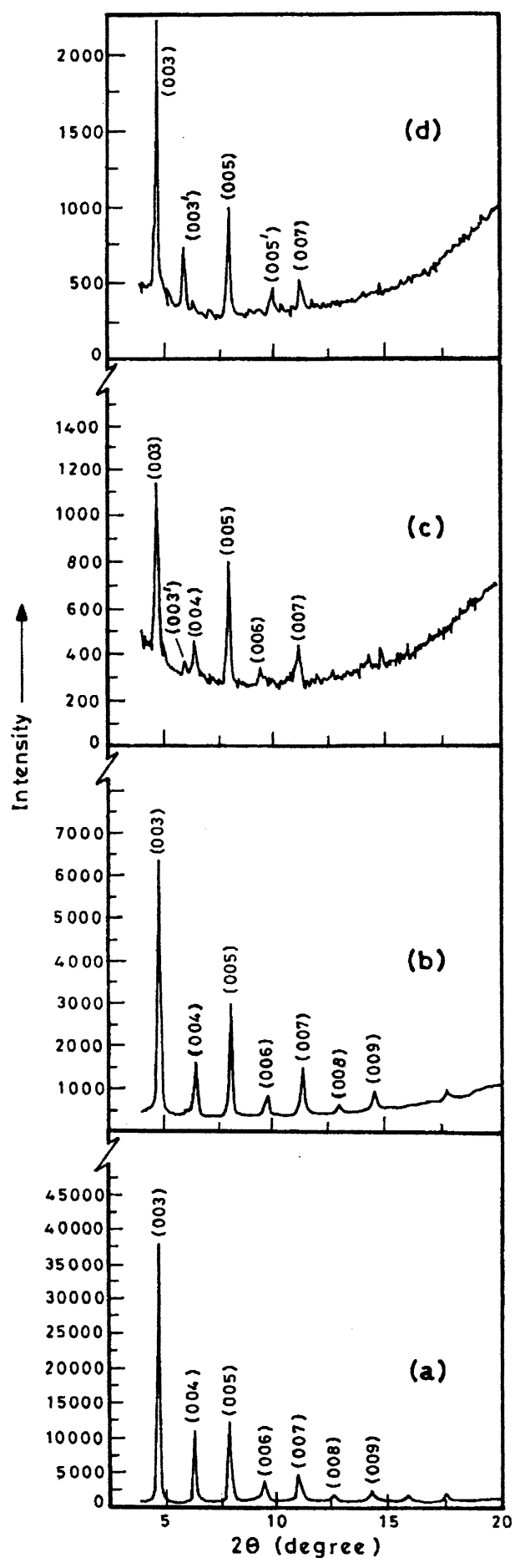

Fig. 3. XR of CdA multilayer in (a) the as-deposited state and after $\mathrm{H}_{2} \mathrm{~S}$ exposure durations for (b) $1 \mathrm{~h}$, (c) $3 \mathrm{~h}$ and (d) $5 \mathrm{~h}$. The evolution of a new layered structure after $3 \mathrm{~h}$ exposure can be seen from the new set of Bragg peaks. 


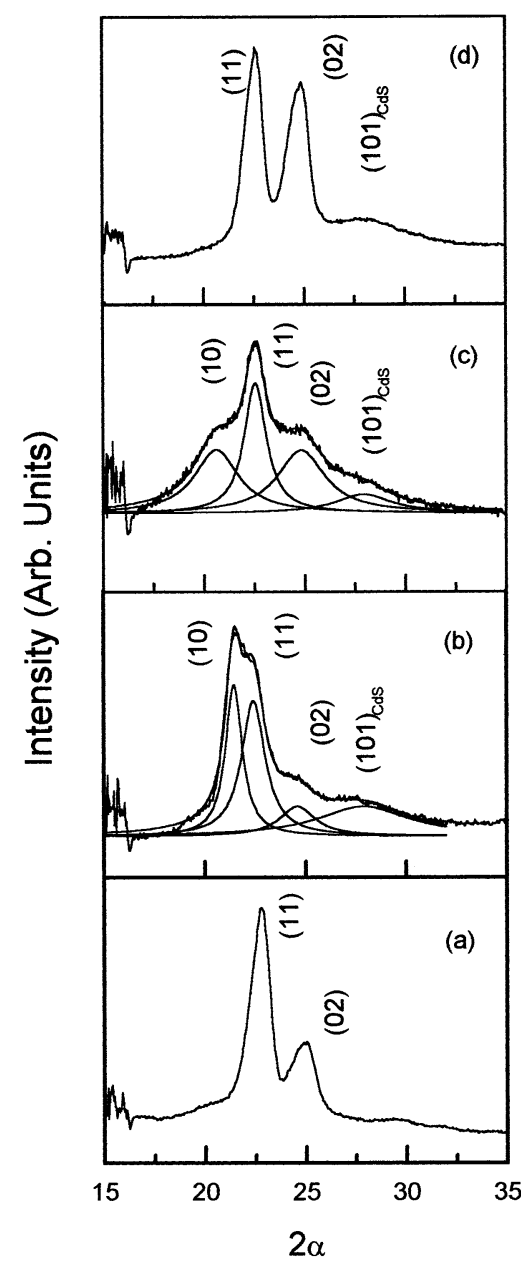

Fig. 4. GIXD pattern of CdA multilayer in (a) the as-deposited state and after $\mathrm{H}_{2} \mathrm{~S}$ exposure durations for (b) $15 \mathrm{~min}$, (c) $3 \mathrm{~h}$ and (d) $5 \mathrm{~h}$, shows two types of in-plane structure for multilayers exposed to $\mathrm{H}_{2} \mathrm{~S}$ for $\sim 3 \mathrm{~h}$. The (10) peak corresponds to hexagonal lattice and (11) and (02) peaks correspond to the centered rectangular lattice.

$R[0,0]$, as proposed by Kitaigorodskii [20] for long chain organic compounds. The GIXD pattern of a 15 min exposed multilayer is shown in Fig. 4(b). The deconvoluted pattern reveals that while the peaks corresponding to the herringbone structure are still present, a new peak appears at $\sim 21.4^{\circ}$. On increasing the exposure to $3 \mathrm{~h}$, this peak decreases in prominence (Fig. 4(c)) and disappears completely after $5 \mathrm{~h}$ of $\mathrm{H}_{2} \mathrm{~S}$ exposure (Fig. 4(d)). It should also be noted that in all the above cases, a broad peak was observed at $\sim 28^{\circ}$. This broad peak is attributed to the (101) reflection from nanoparticulate $\mathrm{CdS}$.

The new peak at $21.4^{\circ}$ can be attributed to (10) reflection of a hexagonal lattice with a lattice constant of $0.48 \mathrm{~nm}$, which closely matches with Kitaigorodskii's $H[0,0]$ packing [20]. The chains in a $H[0,0]$ structure are packed in a hexagonal layer cell and are normal to the layer plane. This result agrees well with XR results, which show that upto $3 \mathrm{~h}$ of $\mathrm{H}_{2} \mathrm{~S}$ exposure, the layered structure corresponds to a bilayer period of $5.5 \mathrm{~nm}$ corresponding to untilted molecules. It is, however, clear that during the first $3 \mathrm{~h}$ of $\mathrm{H}_{2} \mathrm{~S}$ exposure, the molecular packing in the composite multilayer consists of domains of two types of layered structures, a closed packed herringbone and a loosely packed hexagonal. The lateral size of these domains can be determined from the width of the GIXD peaks using the Scherrer relation. The peak widths corresponding to both rectangular and hexagonal packing arrangements for films exposed to $\mathrm{H}_{2} \mathrm{~S}$ for different durations (Fig. 4(b-d)) indicate a finite variation in the corresponding domain sizes, which in all cases are found to be in the range of 5-10 $\mathrm{nm}$.

The appearance of hexagonal layer cell domains in the initial stages of $\mathrm{H}_{2} \mathrm{~S}$ exposure along with the spectroscopic evidence for the formation of $\mathrm{CdS}$ and arachidic acid suggests that the arachidic acid molecules formed are nearly perpendicular to the layer plane in a loosely packed hexagonal layer cell. Continued CdS formation and its possible aggregation in the headgroup region in the vicinity of these domains leads to tilting of these arachidic acid molecules. It is noteworthy that in addition to the tilted molecular domains, the domains with close packed herringbone structure are also present throughout the process during which $\mathrm{CdS}$ formation and aggregation takes place. However, in the case of multilayers exposed for longer durations $(>3 \mathrm{~h}$ ), two types of layered structures with two different bilayer periods but an in-plane structure corresponding to only the herringbone packing is observed. The tilted molecules have a bilayer period of $4.4 \mathrm{~nm}$ corresponding to a tilt angle of $\sim 37^{\circ}$ which is not among the chain tilt angles predicted by Kitaigorodskii [20] for close 
packed structures. Hence, it can be concluded that the observation of two distinct bilayer periods with a single in-plane packing indicates that the tilted molecules have only chain orientational order but no positional order. The difference of $\sim 1.1 \mathrm{~nm}$ between the bilayer periods associated with the untilted and tilted molecular chain domains may be interpreted as follows. If the $\mathrm{CdS}$ nanoclusters with vertical (along the layer normal) dimensions $\sim 1.1 \mathrm{~nm}$ are assumed to grow locally in some headgroup regions only, then this could result in tilting of alkyl chains in the adjacent layers which induces the formation of tilted molecular chain domains in the vicinity of the nanoclusters. Further, the lateral sizes of hexagonal and rectangular domains estimated from GIXD peak widths suggest that $\mathrm{CdS}$ nanoclusters have lateral dimensions in the range of $5-10 \mathrm{~nm}$ and are interspersed with domains of untilted chains with rectangular packing arrangement. It may thus be concluded that the $\mathrm{CdS}$ nanoclusters formed in arachidic acid LB multilayers are quasi two-dimensional in nature, possibly disc shaped with thickness $\sim 1.1 \mathrm{~nm}$ and lateral size $\sim 5-10$ nm.

The shape of CdS nanoclusters formed in the LB matrix has also been studied by Raman spectroscopy. Fig. 5 shows the Raman spectra of CdA multilayers exposed to $\mathrm{H}_{2} \mathrm{~S}$ gas for 15, 30 min and $2 \mathrm{~h}$. All the spectra exhibit a main peak at $\sim 305$ $\mathrm{cm}^{-1}$ corresponding to the LO phonon mode of $\mathrm{CdS}$ along with a low frequency shoulder. The main (LO mode) peak does not show any significant variation in position and shape with exposure duration. This is in contrast to earlier work on CdS nanoclusters, [21-23] where large asymmetric broadening has been observed for cluster sizes less than $10 \mathrm{~nm}$. The full width at half maximum (FWHM) of the LO mode peak in all the three cases lies in the range of $11-14 \mathrm{~cm}^{-1}$, which is slightly larger than the bulk value of $8-10 \mathrm{~cm}^{-1}$. Also, the peak frequency in all the cases shows a very marginal shift from the bulk value of $305 \mathrm{~cm}^{-1}$. The lack of dependence of the frequency and linewidth of LO mode peak on $\mathrm{CdS}$ nanocluster growth may be explained by assuming that the $\mathrm{CdS}$ nanoclusters are constrained to grow two-dimensionally as a thin sheet. In such a case, the confinement effects are predominantly due to the finiteness of thickness of the sheet and it is reasonable that the line-width of the LO mode does not change significantly with growth in lateral direction. Further, if the phonon dispersion along the thickness direction is small, the asymmetric broadening of the LO mode peak may not be substantial. It may also be noted that the smaller clusters having larger energy gap may contribute much less to the Raman signal due to reduced resonance enhancement and this may also be partly responsible for the above behavior.

In order to take into account the shoulder on the low frequency side, the Raman spectra were fitted using two Lorentzians. The fitted spectra are also shown in Fig. 5. In contrast to the behavior of the LO mode peak, the shoulder on the low frequency side shows significant changes with $\mathrm{H}_{2} \mathrm{~S}$ exposure duration. The $15 \mathrm{~min}$ exposed film shows a very broad peak $\sim 290 \mathrm{~cm}^{-1}$. With increase in exposure duration, the broad band

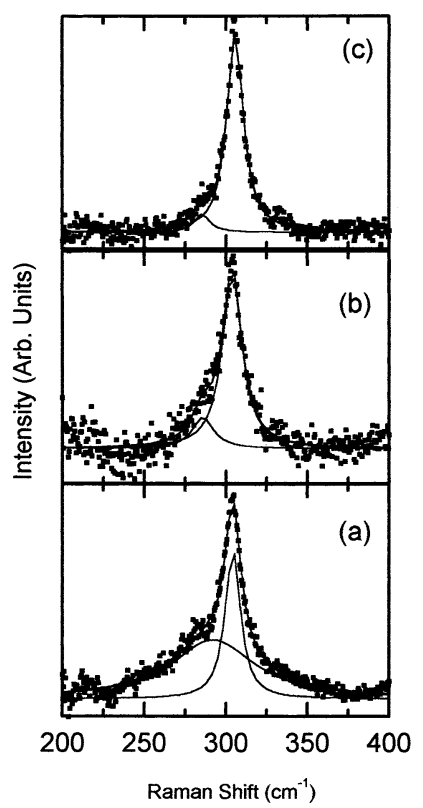

Fig. 5. Raman spectra of CdA multilayers exposed for (a) 15 min, (b) $30 \mathrm{~min}$ and (c) $2 \mathrm{~h}$. The two Lorentzian fit (solid line) and the individual Lorentzians for SO and LO phonon modes (dash lines) are shown. The experimental data shown are after baseline correction. 
slowly starts loosing the width and shifts to $\sim 285$ $\mathrm{cm}^{-1}$ in the cases of $30 \mathrm{~min}$ and $2 \mathrm{~h}$ exposed multilayers. It may be mentioned here that no further change was observed in the nature of the Raman spectra of LB multilayers exposed to $\mathrm{H}_{2} \mathrm{~S}$ for durations as large as $10 \mathrm{~h}$. and the Raman spectrum of $2 \mathrm{~h}$ exposed multilayer (Fig. 5(c)) represents a typical case. Although the origin of the broad band in the $15 \mathrm{~min}$ exposed case (Fig. $5(\mathrm{a})$ ) is not clearly understood, in the later cases (b and c), the shoulder at $285 \mathrm{~cm}^{-1}$ is attributed to surface optic (SO) phonon mode [24]. The persistence of this shoulder and a nearly constant value of $\mathrm{SO} / \mathrm{LO}$ intensity ratio for large exposure durations (upto $10 \mathrm{~h}$ ) is consistent with two-dimensional growth of CdS nanoclusters, i.e. as a thin sheet or disc whose thickness is constrained. This is because for a sphere the surface area to volume $(S / V)$ ratio depends inversely on the radius and drops to zero for large radius, while in the case of a disc the $S / V$ ratio depends inversely on thickness when the radius becomes large and remains non zero for small thickness. In such a situation the $\mathrm{SO} / \mathrm{LO}$ intensity ratio should saturate to a non zero value even if the lateral dimension of the disc becomes large. Thus the behavior of both LO and SO mode Raman peaks suggest that the CdS clusters within LB matrix grow in a two dimensional geometry, in agreement with the structural results.

\section{Conclusions}

The kinetics of CdS nanoclusters formation and their structural organization in the LB matrix has been studied using $\mathrm{CdA}$ multilayers as precursors. It is found that CdS nanoclusters form after exposure to $\mathrm{H}_{2} \mathrm{~S}$ for times as small as $15 \mathrm{~min}$. The formation of $\mathrm{CdS}$, however, saturates in the time period between 2 and $3 \mathrm{~h}$, while the structural changes continue even after $3 \mathrm{~h}$ of exposure. The in-plane molecular organization begins after 15 min and is completed on exposure for $3 \mathrm{~h}$. The vertical layering changes, however, take place only after $\sim 3 \mathrm{~h}$ of exposure. The converted arachidic acid molecules organize into two distinctly separate layered structures with bilayer periods of 5.5 and $4.4 \mathrm{~nm}$. These results suggest that the CdS nanoclusters are formed in the headgroup vicinity of domains with tilted molecular chains, $4.4 \mathrm{~nm}$ periods, and are quasi two-dimensional in nature. The nanoclusters are disc shaped with thickness $\sim 1.1 \mathrm{~nm}$ and lateral size in the range of 5-10 $\mathrm{nm}$. The two-dimensional nature of $\mathrm{CdS}$ nanoclusters is supported by Raman scattering which shows a marginal asymmetric broadening of LO mode peak and a significant presence of the SO mode peak.

\section{Acknowledgements}

Financial assistance from the Department of Science and Technology, Government of India and the Indo-Italian Programme of Co-operation in Science and Technology are gratefully acknowledged.

\section{References}

[1] E.S. Smotkin, C. Lee, A.J. Bard, A. Campion, M.A. Fox, T.E. Mallouk, S.E. Webber, J.M. White, Chem. Phys. Lett. 152 (1988) 265.

[2] B.O. Dabbousi, C.B. Murray, M.F. Rubner, M.G. Bawendi, Chem. Mater. 6 (1994) 216.

[3] F. Grieser, D.N. Furlong, D. Scoberg, I. Ichinose, N. Kimizuka, T. Kunitake, J. Chem. Soc. Faraday Trans. 88 (1992) 2207.

[4] A. Ruaudel-Teixier, J. Leloup, A. Barraud, Mol. Cryst. Liquid Cryst. 134 (1986) 347.

[5] R.S. Urquhart, D.N. Furlong, H. Mansur, F. Grieser, K. Tanata, Y. Okahata, Langmuir 10 (1994) 899.

[6] A. Dhanabalan, H. Kudroli, S.S. Major, S.S. Talwar, Solid State Commun. 9 (1996) 859.

[7] X. Peng, H. Chen, S. Kau, Y. Bai, T. Li, Thin Solid Films 242 (1994) 118.

[8] R. Zhu, G. Min, Y. Wei, H.J. Schmitt, J. Phys. Chem. 96 (1992) 8210.

[9] P. Facci, V. Erokhin, A. Tronin, C. Nicolini, J. Phys. Chem. 98 (1994) 13323.

[10] I. Moriguchi, I. Tanaka, Y. Teraoka, S. Kagawa, J. Chem. Soc. Chem. Commun. (1991) 1401.

[11] J.K. Basu, M.K. Sanyal, Phys. Rev. Lett. 79 (1997) 4617.

[12] F.N. Dultsev, L.L. Sveshnikova, Thin Solid Films 322 (1998) 303.

[13] S. Vitta, T.H. Metzger, S.S. Major, A. Dhanabalan, S.S. Talwar, Langmuir 14 (1998) 1799. 
[14] P. Facci, M.P. Fontana, Solid State Commun. 108 (1998) 5.

[15] Z. Du, Z. Zhang, W. Zhao, Z. Zhu, J. Zhang, Z. Jin, T. Li, Thin Solid Films 210/211 (1992) 404.

[16] T. Yamaki, K. Asai, K. Ishigure, Chem. Phys. Lett. 273 (1997) 376.

[17] L.E. Brus, J. Chem. Phys. 80 (1984) 4403.

[18] M.V. Ramakrishna, R.A. Friesner, J. Chem. Phys. 95 (1991) 8301.
[19] P. Tippmann-Krayer, R.M. Kenn, H. Möhwald, Thin Solid Films 210/211 (1992) 577.

[20] A.I. Kitaigorodskii, Organic Chemical Crystallography, Consultant Bureau, NewYork, 1961.

[21] A.G. Rolo, L.G. Vieira, M.J.M. Gomes, J.L. Ribeiro, M.S. Belsley, M.P. dosSantos, Thin Solid Films 312 (1998) 348.

[22] A. Roy, A.K. Sood, Solid State Commun. 97 (1996) 97.

[23] A. Ingale, K.C. Rustagi, Phys. Rev. B 52 (1998) 7189.

[24] M.C. Klein, F. Hache, D. Ricard, C. Flytzanis, Phys. Rev. B 42 (1990) 11123. 